\title{
A Hybrid Approach to Estimating Error Covariances in Variational Data Assimilation
}

\author{
Haiyan Cheng, Mohamed Jardak, Mihai Alexe, Adrian Sandu *,1 \\ Computational Science Laboratory \\ Department of Computer Science \\ Virginia Polytechnic Institute and State University \\ 2201 Knowledgeworks II, 2202 Kraft Drive, Blacksburg, VA 24060, USA \\ Email: hcheng04@vt.edu, mjardak@vt.edu, mihai@vt.edu, sandu@cs.vt.edu
}

\begin{abstract}
Data Assimilation (DA) involves the combination of observational data with the underlying dynamical principles governing the system under observation. In this work we combine the advantages of the two prominent advanced DA systems, the 4D-Var and the ensemble methods. The proposed method consists of identifying the subspace spanned by the major 4D-Var error reduction directions. These directions are then removed from the background covariance through a Galerkin-type projection. This generates an updated error covariance information at both end points of an assimilation window. The error covariance information is updated between assimilation windows to capture the "error of the day". Numerical results using our new hybrid approach on a nonlinear model demonstrate how the background covariance matrix leads to an error covariance update that improves the 4D-Var DA results.
\end{abstract}

Key words: Data Assimilation, Ensemble Kalman Filter, Hybrid Method, Error Covariance

\section{Introduction}

Data Assimilation (DA) is a relatively novel and versatile multidisciplinary methodology. Given a consistent numerical model for a complex time-dependent system, and a set of observations of the model state taken at various time moments, DA allows us to combine these two sources of information to obtain a state approximation, which is, in a well defined statistical sense, the "best" estimate of the true model state.

DA comprises three types of methods: interpolation, variational, and sequential methods $[6,8$, $16,27,29]$. In the first method, the measurements are interpolated from the points of observation onto the grid points. The interpolation can be weighted by the statistics of the observations. While simple to implement, this approach is not justified by any physical arguments. The variational and sequential data assimilation methods fit into the framework of estimation theory. Sequential DA features the Kalman filter, as described in Kalman [9] and Jazwinski [17], the Ensemble Kalman

\footnotetext{
This work has been partially supported by NSF CCF-0515170, NSF CCF-0635194, and NASA ROSES-2005 AIST project led by Dr. Meemory Lee.

*Corresponding author. Email: sandu@cs.vt.edu. Phone: +1-540-231-2193. Fax: +1-540-231-9218. 
Filter (EnKF) of Evensen [13, 14, 12, 11], as well as several types of particle filters (see, e.g., Doucet $[1,2]$, Arulampalam [31], and Berliner [18]). The main drawback of sequential data assimilation stems comes from the fact that the covariance matrices of the state variables have huge dimensions for operational models.

The variational methods were introduced by Sasaki [33]. These methods consider the equations governing the flow as constraints, and the problem is closed by using a variational principle, e.g., the minimization of the discrepancy between the model and the observations. This problem fits into the framework of optimal control theory [24], as it was proposed by Le Dimet [20], Le Dimet and Talagrand [21], and Courtier and Talagrand [7]. The major drawback of the variational methods is in assuming the background error covariances are static, nearly homogeneous and isotropic. This has been pointed out by Parrish and Derber [30], Courtier et al. [29], Cohn et al. [34], and Lorenc [5]. The ensemble methods provides a alternative to variational data assimilation. The EnKF estimates the background error covariances from an ensemble of short-term forecasts, as described in the work of Houtkamer and Mitchell [19], and Anderson [3]. Although sequential methods and variational methods are implemented separately, some connections between them have long been known. In fact, for a perfect model, with the same input data (initial background, background covariance, distribution of observations, and observation covariance), both the 4D-Var and the Kalman filter yield the same analysis at the end of the assimilation window [35, 36]. This equivalence between sequential and variational methods provides a possibility of combining the two approaches, leading to powerful hybrid DA algorithms.

There have been several recent research efforts in this direction. The 3DVar/EnKF of Hamill and Snyder [26] is a hybrid scheme blending the 3D-Var and the Ensemble Kalman filter. It utilizes the ensemble framework to propagate the estimation statistics of the model. However, it does not fully exploit the smoothing characteristics of 4D-Var. The Ensemble Kalman Smoother (EnKS) of Evensen and van Leeuwen [10] recomputes a new analysis for all recent measurements upon the receipt of each new observations. This approach is computationally intractable for real world problems. Zhang et al. [38] devised the Ensemble 4D-Var (E4DVAR). E4DVAR is essentially constructed as a feedback loop between 4D-Var and the EnKF method. The ensemble mean in EnKF is periodically replaced by the $4 \mathrm{D}$-Var analysis, and the covariance matrix built from the EnKF ensemble provides the background information in 4D-Var. Bewley et al. [4] proposed the Ensemble Variational Estimation (EnVE) method. EnVE is a coupled hybrid of EnKF/EnKS and $4 \mathrm{D}$-Var. It leverages the nonlinear statistical propagation properties of the EnKF/EnKS to initialize and properly define an consistent variational iteration similar to 4D-Var.

This research work proposes a novel hybrid method to generate covariance estimates at both endpoints of the assimilation window. Our method is the first approach to explicitly consider the error components removed by the numerical optimization method in $4 \mathrm{D}$-Var. Central to this hybrid DA method is the construction of a projection operator $\mathcal{P}$. We first build the subspace spanned by the first search directions explored by the $4 \mathrm{D}$-Var optimizer. The operator $\mathcal{P}$ is then defined as the orthogonal projector onto the orthogonal complement of this subspace. Next, we use the background covariance $B$ to construct an ensemble of perturbations around the given background state. This ensemble has mean zero and covariance $B$. The next step is the construction of hybrid ensemble, by adding the projected background ensemble perturbations to the 4D-Var optimum iterate. The hybrid covariance is then assembled from the hybrid ensemble members. Two different approaches allow us to update the error covariance information between windows. We can propagate each hybrid ensemble member through the model to the next assimilation window (hy- 
brid propagation). Alternatively, we can apply the projection operator on the background ensemble perturbations at the final time of the assimilation window (hybrid projection). The latter approach can be essentially thought of as a Galerkin-like projection of the background covariance $B$ onto the ortogonal complement of the space spanned by the leading 4D-Var error reduction directions. The new background covariance estimate can be constructed as a convex linear combination of the static background $B$ and the hybrid covariance. The numerical experiments in section 4 show that the hybrid covariance estimate is superior to the static background. Moreover, the hybrid covariance update leads to considerable improvement in the quality of the model forecast.

The rest of this paper is structured as follows. Section 2 discusses the mathematical foundation of the 4D-Var method. In section 3 we present a step by step description of our hybrid method. Next, the hybrid DA scheme is tested on a version of the the Lorenz 96 model [25] in section 4. Finally, section 5 contains the summary and conclusions, along with directions for further research.

\section{Mathematical Background on 4D-Var}

Consider a time-dependent model given by the initial value problem

$$
\begin{cases}\frac{d x}{d t} & =\mathbb{M}(t, x) \quad t_{0} \leq t \leq t_{F} \\ x\left(t_{0}\right) & =x_{0}\end{cases}
$$

Let $\mathcal{M}$ be the discrete solution operator for the model (1). The discrete approximation to the exact model state at time $t=t_{i}$ is $x_{i} \in \mathbb{R}^{n}$ :

$$
x_{i}=\mathcal{M}_{t_{0} \rightarrow t_{i}} x_{0} .
$$

In the following we will denote by $t_{0}$ and $t_{F}$ the start time and the end time of a $4 \mathrm{D}$-Var assimilation window, respectively.

Given an a priori forecast error covariance matrix $B_{t_{0}} \in \mathbb{R}^{n \times n}$, a background model state $x_{0}^{b} \in \mathbb{R}^{n}$ at $t_{0}$, and a set of noisy observations $y_{i} \in \mathbb{R}^{m}$ at selected times $t_{i}$, the discrete $4 \mathrm{D}$ Var data assimilation problem consists of determining the maximum likelihood initial model state $x^{a}\left(t_{0}\right)$ as the minimizer of the following cost functional:

$$
\begin{aligned}
\mathcal{J}\left(x_{0}\right) & =\mathcal{J}^{\mathrm{b}}+\mathcal{J}^{\mathrm{o}} \\
& =\left(x_{0}-x_{0}^{b}\right)^{T} B_{t_{0}}^{-1}\left(x_{0}-x_{0}^{b}\right)+\sum_{i=1}^{N_{\text {obs }}}\left(\mathcal{H}_{i} x_{i}-y_{i}\right)^{T} R_{i}^{-1}\left(\mathcal{H}_{j} x_{i}-y_{i}\right) .
\end{aligned}
$$

The $\mathcal{H}_{i}$ are observation operators that map the state variables $x_{i} \approx x\left(t_{i}\right)$ to the observation space:

$$
y_{i}=\mathcal{H}_{i}\left(x_{i}\right)
$$

$\mathcal{J}^{\mathrm{b}}$ represents the departure from the background value $x^{b}$, while $\mathcal{J}^{\mathrm{o}}$ measures the misfit between the model prediction and the observations.

Current 4D-Var assimilation studies use the same background covariance matrices for each assimilation window. Ideally however, this background information should be updated periodically to capture the system dynamics. Using 4D-Var with an improved background covariance would lead to a higher quality forecast with a larger range of validity. 
Knowledge of the a posteriori error statistics at the endpoints of the $4 \mathrm{D}$-Var window can be very valuable. Suppose we compute a good estimate $A_{t_{F}}$ of the a posteriori covariance at the end of the assimilation window. This is used as the background covariance for the next assimilation window, allowing us to account for the errors of the day, and ultimately improve the quality of our forecast.

\section{The Hybrid Method}

4D-Var experiments usually use a static prescribed background error covariance at the beginning of each assimilation window. This matrix is built using statistical information inferred from the background state, the available observations, and the model dynamics. The drawback of this approach is that an accurate analysis is only guaranteed within each window. The ideal DA method should account for the "errors of the day", by updating the background error covariance matrix between assimilation windows.

Our goal is to estimate the analysis error covariances $A_{t_{0}}$ and $A_{t_{F}}$, so that the approximated $A_{t_{F}}$ can be used as the background covariance in the next assimilation window. Note that $4 \mathrm{D}$-Var by itself does not yield any covariance information. Second order adjoint models $[22,37]$ can be used to obtain information about the eigendirections of $\nabla^{2} \mathcal{J}$. The inverse Hessian $\left(\nabla^{2} \mathcal{J}\right)^{-1}$ is known to approximate the a posteriori error covariance matrix at $t_{0}$ [15]. Thus, the eigenvectors corresponding to the smallest Hessian eigenvalues will give the directions of maximum error growth in the dynamical model (1).

Our method makes use of three ensembles. The background ensemble is generated using the error statistics given by $B_{t_{0}}$, and each of its members is propagated to $t_{F}$. Then, through multiple $4 \mathrm{D}$-Var runs, each of which is started from one background ensemble member, we compute an ensemble of analysis states at $t_{0}$. This reference analysis ensemble gives the reference error covariance matrices at the initial and final assimilation times. The 4D-Var analysis is started from the background state $x_{0}^{b}$. The vectors in this subspace are the directions of maximum error growth that are canceled by 4D-Var during the first optimization iterations. A hybrid ensemble is generated by projecting the background ensemble perturbations out of the space spanned by the first several 4D-Var increments. The hybrid covariance is then assembled from the hybrid ensemble members. This is done at $t_{0}$ and $t_{F}$. We assess the quality of the hybrid covariance approximation through an eigenvalue comparison against the reference analysis covariance.

In the following we give a detailed description of our algorithm.

\subsection{The Background Ensemble}

- At $t=t_{0}$.

Suppose we are given the background state $x^{b}\left(t_{0}\right) \in \mathbb{R}^{n}$, the background covariance matrix $B_{0} \in \mathbb{R}^{n \times n}$, a set of observations $y_{j} \in \mathbb{R}^{m}$ taken at times $t_{j}$, for $j=1, \ldots, N_{\text {obs }}$, and the observation covariances $R_{j} \in \mathbb{R}^{m \times m}$.

1. Generate a set of $N_{e}$ normally distributed perturbations with mean zero and covariance $B_{t_{0}}$ :

$$
\Delta x_{i}^{b} \in \mathcal{N}\left(0, B_{t_{0}}\right), \quad i=1, \ldots N_{e} .
$$

2. Construct a background ensemble of size $N_{e}$ :

$$
x_{i}^{b}\left(t_{0}\right)=x^{b}\left(t_{0}\right)+\Delta x_{i}^{b}, \quad i=1, \ldots, N_{e} .
$$


- At $t=t_{F}$.

1. The background ensemble members at $t_{0}$ are propagated by running the model up to $t_{F}$. We obtain a new ensemble

$$
\left\{x_{1}^{b}\left(t_{F}\right), x_{2}^{b}\left(t_{F}\right), \ldots, x_{N_{e}}^{b}\left(t_{F}\right)\right\},
$$

where

$$
x_{i}^{b}\left(t_{F}\right)=\mathcal{M}_{t_{0} \rightarrow t_{F}} x_{i}^{b}\left(t_{0}\right), \quad i=1, \ldots, N_{e} .
$$

2. Compute the mean $x^{b}\left(t_{F}\right)$ and covariance $B_{t_{F}}$ for this ensemble.

\subsection{The Hybrid Ensemble}

- At $t=t_{0}$.

Starting from $x^{b}\left(t_{0}\right)$, the numerical optimization routine used by $4 \mathrm{D}$-Var generates a sequence of iterates $x^{(i)}, i=1, \ldots k$. Here $k$ is a truncation index, in that we retain only the first $k$ iterates, with $k \ll n$. This strategy is consistent with the observation that the reduction in the cost function is fastest during the initial iterations of the optimizer. This phenomenon has been consistently observed in practice (see, e.g., [23, 28, 32, 40]).

1. Let $x_{0}^{(i)}$ denotes the $i$-th iterate computed by the optimizer, and denote by $\mathcal{S}_{t_{0}}$ the space spanned by the normalized 4D-Var increments

$$
\mathcal{S}_{t_{0}}=\left[\frac{x_{0}^{(i)}-x_{0}^{(i-1)}}{\left\|x_{0}^{(i)}-x_{0}^{(i-1)}\right\|}\right]_{i=1, \ldots, k},
$$

with $x_{0}^{(0)} \equiv x^{b}\left(t_{0}\right)$.

2. Using the singular value decomposition (SVD) of $\mathcal{S}_{t_{0}}$

$$
\mathcal{S}_{t_{0}}=U \Sigma V^{T}
$$

we retain only the right singular vectors $u_{i}, i=1, \ldots, l$ that correspond to the largest $l$ singular values $\sigma_{1}, \ldots, \sigma_{l}$, where

$$
\frac{\sigma_{k}}{\sigma_{1}}<0.1, \forall k>l
$$

Let

$$
U_{t_{0}}=\left[\begin{array}{llll}
u_{0}^{1} & u_{0}^{2} & \ldots & u_{0}^{l}
\end{array}\right] .
$$

3. The orthogonal projector onto the orthogonal complement of $U_{t_{0}}$ is defined as

$$
\mathcal{P}_{t_{0}}=I-U_{t_{0}} U_{t_{0}}^{T} .
$$

4. Project the ensemble perturbations $\Delta x_{i}^{b}$ out of the space $U_{t_{0}}$, and generate the hybrid ensemble around the optimal 4D-Var iterate $x_{0}^{(k)}$ :

$$
\begin{aligned}
\Delta x_{i}^{h}\left(t_{0}\right) & =\mathcal{P}_{t_{0}} \Delta x_{i}^{b}\left(t_{0}\right) \\
x_{i}^{h}\left(t_{0}\right) & =x_{0}^{(k)}+\Delta x_{i}^{h}\left(t_{0}\right) . \\
5 &
\end{aligned}
$$


5. Compute the covariance $A_{t_{0}}^{h}$ of the hybrid ensemble. The background perturbations (5) are normally distributed with zero mean, and covariance $B_{t_{0}}$. Since $\mathcal{P}_{t_{0}}$ is linear, the hybrid ensemble at $t_{0}$ has mean $x_{0}^{(k)}$ :

$$
\mathbb{E}\left[x_{i}^{h}\right]=x_{0}^{(k)}+\mathcal{P}_{t_{0}} \mathbb{E}\left[x_{i}^{b}\right]=x_{0}^{(k)},
$$

and covariance

$$
A_{t_{0}}^{h}=\mathcal{P}_{t_{0}} B_{t_{0}} \mathcal{P}_{t_{0}}
$$

6. Calculate the eigenvalues $\left\{\rho_{1}, \rho_{2}, \ldots, \rho_{N_{e}}\right\}$, and eigenvectors $\left\{w_{1}, w_{2}, \ldots, w_{N_{e}}\right\}$ of $A_{t_{0}}^{h}$.

- At $t=t_{F}$ :

1. Let

$$
\mathcal{S}_{t_{F}}=\left[\frac{x_{F}^{(i)}-x_{F}^{(i-1)}}{\left\|x_{F}^{(i)}-x_{F}^{(i-1)}\right\|}\right]_{i=1, \ldots, k},
$$

where $x_{F}^{0} \equiv x^{b}\left(t_{F}\right)$. Note that all of the iterates

$$
x_{F}^{(i)}=\mathcal{M}_{t_{0} \rightarrow t_{F}} x_{0}^{(i)}
$$

are readily available, as they have been computed during the $4 \mathrm{D}$-Var iterations started from $x^{b}\left(t_{0}\right)$.

2. We discard all but the first $l$ left singular vectors $u_{F}^{i}(i=1, \ldots, l)$ of $\mathcal{S}_{t_{F}}$, using the SVD and equation (11). Let

$$
U_{t_{0}}=\left[\begin{array}{llll}
u_{F}^{1} & u_{F}^{2} & \ldots & u_{F}^{l}
\end{array}\right] .
$$

Then

$$
\mathcal{P}_{t_{F}}=I-U_{t_{F}} U_{t_{F}}^{T}
$$

is the orthogonal projector out of $\mathcal{S}_{t_{F}}$. We perform an orthogonal projection of the background ensemble perturbations

$$
\Delta x_{i}^{b}\left(t_{F}\right)=x^{b}\left(t_{F}\right)-x_{i}^{b}\left(t_{F}\right)
$$

on the orthogonal complement of $\mathcal{S}_{t_{F}}$, to generate the hybrid ensemble members at $t_{F}$ :

$$
\begin{aligned}
\Delta x_{i}^{h}\left(t_{F}\right) & =\mathcal{P}_{t_{F}} \Delta x_{i}^{b}\left(t_{F}\right) \\
x_{i}^{h}\left(t_{F}\right) & =x_{F}^{(k)}+\Delta x_{i}^{h}\left(t_{F}\right), \quad i=1, \ldots, N_{e} .
\end{aligned}
$$

The background ensemble perturbations at $t_{F}$ are normally distributed with mean zero, and covariance $B_{t_{F}}$. Hence, the hybrid ensemble at $t_{F}$ is also Gaussian, with mean $x_{F}^{(k)}$ (the iterate closest to the optimal value):

$$
\mathbb{E}\left[x_{i}^{h}\left(t_{F}\right)\right]=x_{F}^{(k)}+\mathcal{P}_{t_{F}} \mathbb{E}\left[\Delta x_{i}^{b}\left(t_{F}\right)\right],
$$

and covariance

$$
A_{t_{F}}^{h}=\mathcal{P}_{t_{F}} B_{t_{F}} \mathcal{P}_{t_{F}}
$$


Alternatively, the hybrid ensemble members can be propagated via the model from $t_{0}$ to $t_{F}$. This approach has a higher computational cost than the final time projection algorithm, and it leads to a different hybrid ensemble:

$$
\widetilde{x}_{i}^{h}\left(t_{F}\right)=\mathcal{M}_{t_{0} \rightarrow t_{F}} x_{i}^{h}\left(t_{0}\right), \quad i=1, \ldots, N_{e} .
$$

Our experiments with the Lorenz 96 model [25] indicate that the final time projection (22) leads to a more accurate a posteriori covariance estimate than the propagation method (25). Section 4 describes the numerical experiments in detail.

\subsection{The Analysis Reference Ensemble}

- At $t=t_{0}$ :

Consider the initial time background ensemble (6). Run 4D-Var starting from each ensemble member $x_{i}^{b}\left(t_{0}\right)$, using

$$
y_{j}=\mathcal{H}\left(\mathcal{M}_{t_{0} \rightarrow t_{j}} x_{j}\right)+\epsilon_{i j}, j=1, \ldots, N_{\text {obs }},
$$

where $\epsilon_{i j} \in \mathcal{N}\left(0, R_{j}\right), R_{j} \in \mathbb{R}^{m \times m}$. This generates a new ensemble of analysis states at the initial assimilation time, henceforth referred to as the analysis reference ensemble at $t_{0}$.

1. Compute the mean $x^{a}\left(t_{0}\right)$ and the covariance $A_{t_{0}}^{a}$ of the analysis reference ensemble:

$$
\begin{aligned}
x^{a}\left(t_{0}\right) & =\frac{1}{N_{e}} \sum_{i=1}^{N_{e}} x_{i}^{a}\left(t_{0}\right) \\
A_{t_{0}}^{a} & =\frac{1}{N_{e}-1} \sum_{i=1}^{N_{e}}\left[x_{i}^{a}\left(t_{0}\right)-x^{a}\left(t_{0}\right)\right]\left[x_{i}^{a}\left(t_{0}\right)-x^{a}\left(t_{0}\right)\right]^{T} .
\end{aligned}
$$

2. Compute the eigenvalue decomposition of $A_{t_{0}}^{a}$ to obtain the eigenvalues $\left\{\mu_{1}, \mu_{2}, \ldots, \mu_{n}\right\}$ and the corresponding orthonormal eigenvectors $\left\{v_{1}, v_{2}, \ldots, v_{n}\right\}$.

- At time $t_{F}$.

1. The analysis reference ensemble members at $t_{0}$ are propagated by the model up to $t_{F}$, to get

$$
\left\{x_{1}^{a}\left(t_{F}\right), x_{2}^{a}\left(t_{F}\right), \ldots, x_{N_{e}}^{a}\left(t_{F}\right)\right\} .
$$

2. Let $x^{a}\left(t_{F}\right)$ be the mean, and $A_{t_{F}}^{a}$ the covariance of this ensemble.

To assess the results of the hybrid method, compare the hybrid covariance estimates $A_{t_{0}}^{h}$ and $A_{t_{F}}^{h}$ against the analysis reference covariances $\left(A_{t_{0}}^{a}, A_{t_{F}}^{a}\right)$, and the background covariances $\left(B_{t_{0}}\right.$, $\left.B_{t_{F}}\right)$. Section 4 discusses in details the results obtained for a Lorenz-96 system with 40 variables. 


\subsection{The Computational Cost of the Hybrid DA Method}

The two most expensive steps of the hybrid DA method are:

- the forward propagation of the background ensemble members (6) through the nonlinear model, from $t_{0}$ to $t_{F}$, and

- the computation of $4 \mathrm{D}$-Var iterates (9) starting from $x^{b}\left(t_{0}\right)$.

However, note that these two phases are independent. Thus, they can be performed in parallel on a multiprocessor computer, substantially decreasing the computational cost of the hybrid method. Once the final time background ensemble (7), and the optimization iterates (9-17) have been computed, the hybrid projection method can be readily applied. The only additional cost is incurred when computing the SVD of $\mathcal{S}_{t_{0}}$ and $\mathcal{S}_{t_{F}}$. The hybrid propagation approach has a higher computational cost, since it needs to propagate the hybrid ensemble members from $t_{0}$ to $t_{F}$. Each hybrid ensemble member can be evolved independently of the rest, but there is no source of parallelism beyond this level. All the hybrid ensemble members at $t_{0}$ have to be available before the hybrid propagation method can compute $A_{t_{F}}^{h}$.

\section{Numerical Experiments}

We now test our hybrid DA strategy on the Lorenz 96 model [25]. This dynamical model is described by the following set of equations:

$$
\frac{d x_{j}}{d t}=-x_{j-1}\left(x_{j-2}-x_{j+1}-x_{j}\right)+F, \quad j=1, \ldots 40,
$$

with periodic boundary conditions: $x_{0}=x_{40}, x_{-1}=x_{39}$.

The background covariance $B_{t_{0}}$ is constructed from a $3 \%$ perturbation of the initial state, and a correlation distance of $L=1.5$ :

$$
B_{t_{0}}(i, j)=\sigma_{i} \cdot \sigma_{j} \cdot \exp \left(-\frac{d^{2}}{L^{2}}\right), i, j=1, \ldots, 40 .
$$

We take into account the periodic boundaries when computing $d=|i-j|$. The observation covariance matrix is diagonal from a $\rho=1 \%$ perturbation from the mean observation values. The observation operator $\mathcal{H}$ captures only a subset of 30 model states, which include every other states from the first 20 states plus the last 20 states. The length of one assimilation window is set to be 1 time unit. Our implementation of 4D-Var makes use of the L-BFGS routine of Zhu et al. [39], the de-facto "gold standard" of gradient-based optimizers used in data assimilation studies. Finally, the ensemble 4D-Var reference solution is generated using 1000 ensemble members.

The simulation time spans two consecutive DA windows. We designates by $B_{t_{0}}$ the background covariance matrix at $t_{0}$, the start time of the first DA window. First, we generate an approximation $A_{t_{1}}^{h}$ to the a posteriori error covariance matrix at $t_{1}$, the end time point in the first assimilation window. This hybrid covariance is then used as the background information in the second assimilation window, which ends at $t_{2}$. It is shown that the hybrid covariance is a good approximation to the reference covariance at $t_{1}$ (computed using an ensemble of $4 \mathrm{D}$-Var analysis). Moreover, the use of $A_{t_{1}}^{h}$ as background information in the second window leads to a considerable improvement in the quality of the forecast. 


\subsection{The first assimilation window}

Figure (2) shows the eigenvalues of the a posteriori covariance approximations at $t_{0}$ and $t_{1}$. We compute two approximations of the hybrid covariance at $t_{1}$. First, the background ensemble perturbations at $t_{1}$ are projected out of the subspace of $4 \mathrm{D}$-Var increments $S_{t_{1}}$, as in (22). For the second approximation, the hybrid ensemble members computed at $t_{0}$ are propagated through the model (28) up to time $t_{1}$.

It is seen that the hybrid method leads to better approximations of the reference covariance than the background, both at $t_{0}$ and at $t_{1}$. The agreement between the hybrid and the analysis covariance is even better at $t_{1}$ than at $t_{0}$. We conjecture that, for this particular model, the error directions that are not taken into account by $4 \mathrm{D}$-Var during the optimization procedure are reduced by the system dynamics. Furthermore, as expected, the projection of the background ensemble at $t_{1}$ onto the orthogonal complement of $\mathcal{S}_{t_{1}}$ yields results superior to those obtained when evolving the hybrid ensemble from $t_{0}$ to $t_{1}$. Note that the $4 \mathrm{D}$-Var as well as the ensemble runs can be performed in parallel on a multiprocessor computer. This leads to a significant decrease in the CPU time spent in the covariance approximation procedure, over the case of fully serial optimization and model evaluations.

\subsection{The second assimilation window}

At the beginning of the second time window, the background error covariance matrix $B_{t_{1}}$ is updated to account for the model dynamics, as follows:

$$
B_{t_{1}}=\alpha B_{t_{0}}+(1-\alpha) A_{t_{1}}^{h} .
$$

Here $B_{t_{0}}$ is the background covariance for the first window, and $A_{t_{1}}^{h}$ is the a posteriori error covariance computed by the hybrid method. For our particular experiment, $\alpha=0.1$.

Figure 3 shows the covariance approximations at $t_{1}$ (now the beginning of second window). The hybrid DA yields a covariance that is more accurate than the one stemming from the background ensemble.

In order to assess the impact of the improved background covariance matrix on the model forecast, we run the $4 \mathrm{D}$-Var in the second window using

- the constant background covariance from the first window $B_{t_{0}}$, and

- the updated covariance $B_{t_{1}}$ as defined in (30).

The root mean square error(RMSE) of the two optimal trajectories inside $\left[t_{1}, t_{2}\right]$ is defined by

$$
R M S E=\sqrt{\frac{1}{N} \sum_{i=1}^{N} \frac{\left\|x^{t}\left(t_{i}\right)-x_{i}\right\|_{2}^{2}}{\left\|x^{t}\left(t_{i}\right)\right\|_{2}^{2}}}
$$

where $x^{t}\left(t_{i}\right)$ is the "true" solution at $t_{i}$, and $x_{i}=\mathcal{M}_{t_{1} \rightarrow t_{i}} x\left(t_{1}\right)$.

The two optimal trajectories were compared at $N=100$ equidistant time points in $\left[t_{1}, t_{2}\right]$. The RMSE of the analysis computed using $B_{t_{0}}$ is 0.0028 . The updated hybrid covariance reduces the RMSE to 0.0011. This two-fold reduction in the error suggests that the hybrid covariance matrix does a good job of capturing the main components of the "error of the day" (i.e., the error accumulated during the first assimilation window), which leads to a more accurate forecast. 


\section{Conclusion}

Recently, considerable research efforts have been put into the development of variational DA procedure that updates the background covariance matrices such that the specific flow dynamics (hence, the "errors of the day") can be taken into account. This paper proposes a hybrid DA method that yields an approximation of the a posteriori error covariance matrix. This hybrid covariance is built by removing the most significant error directions explored by 4D-Var (during the first few optimization iterations) from a set of background ensemble perturbations. The projection operator is easy to construct at both endpoints of an assimilation window because the basis vectors are provided "for free" by the 4D-Var process. Both the $4 \mathrm{D}$-Var and the background ensemble runs can be performed in parallel on a multiprocessor machine. This significantly lowers the computational cost of the hybrid method. The only additional cost comes from the background ensemble projection at the window end points (for the hybrid projection strategy), or from the propagation of the hybrid ensemble from the beginning to the end of the window, in the case of the hybrid propagation approach.

We demonstrate the hybrid DA strategy on the Lorenz-96 system. The hybrid approach is found to yield an a posteriori covariance approximation which is considerably superior to that inferred from the background state information. The use of this hybrid covariance in the next assimilation window results in an optimal trajectory whose RMSE is two times smaller than that of the analysis obtained when reusing the constant background covariance. Hence, the hybrid projection method correctly accounts for the "errors of the day", and is expected to improve the quality of the model forecast.

\section{References}

[1] Doucet. A., de Freitas. J. F., and Gordon. N. J. An introduction to sequential Monte Carlo methods. Sequential Monte Carlo methods in practice, 2001.

[2] Doucet. A., Godsill. S., and Andrieu. C. On sequential Monte Carlo sampling methods for Bayesian filtering. Statistics and Computing, 10(30):197-208, 2000.

[3] J. L. Anderson. An ensemble adjustment Kalman filter for data assimilation. Monthly Weather Review, 129:28842903, 2001.

[4] T. Bewley, J. Cessna, and C. Colburn. EnVE: A consistent hybrid ensemble/variational estimation strategy for multiscale uncertain systems. Tellus, 2009.

[5] Lorenc A. C. The potential of the Ensemble Kalman Filter for NWP-A comparison with the 4D-VAR. Quarterly Journal of Royal Meterology Society, 129:3183-3203, 2003.

[6] S. E. Cohn. An introduction to estimation theory. Journal of the Meteorological Society of Japan, 75:257-288, 1997.

[7] P. Courtier and O. Talagrand. Variational assimilation of meteorological observations with the adjoint equations. Part 2: Numerical results. Quarterly Journal of Royal Meterology Society, 113:1329-1347, 1987.

[8] R. Daley. Atmospheric Data Analysis. Cambridge University Press, 1991.

[9] Kalman. R. E. A new approach to linear filtering and prediction problems. Transaction of the ASME -Journal of Basic Engineering, 82(Series D):35-45, 1960.

[10] G. Evensen and P.J. van Leeuwen. An ensemble Kalman smoother for nonlinear dynamics. Monthly Weather Review, 128:1852-1867, 2000.

[11] Burgers. G., van Leeuwen. P. J., and Evensen. G. Analysis scheme in the ensemble Kalman filter. Monthly Weather Review, 126:1719-1724, 1998.

[12] Evensen. G. Sequential data assimilation with a nonlinear quasi-geostrophic model using Monte Carlo methods to forecast error statistics. Journal of Geophysics Research, 99 (C5 10):108-129, 1994.

[13] Evensen. G. The ensemble Kalman filter: theoretical formulation and practical implementation. Ocean Dynamics, 53:343-367, 2003.

[14] Evensen. G. Data Assimilation: The ensemble Kalman filter. Springer, Berlin, 2007. 
[15] I. Yu Gejadze, F.-X. Le Dimet, and V. Shutyaev. On analysis error covariances in variational data assimilation. SIAM Journal on Scientific Computing, 30(4):1847-1874, 2008.

[16] M. Ghil, K. Ide, A. F. Bennett, P. Courtier, M. Kimoto, and N. Sato. Data assimilation in meteorology and oceanography: Theory and practice. Journal of the Meteorological Society of Japan, 75:257-288, 1997.

[17] A. H. Jazwinski. Stochastic processes and filtering theory. Academic Press: New York, 1970.

[18] Berliner. M. L. and Wikle. C. K. A Bayesian tutorial for data assimilation. Physica D, 30:1-16, 2007.

[19] Houtekamer. P. L. and Mitchell. H. L. A sequential ensemble Kalman filter for atmospheric data assimilation. American Meteorological Society, 129(1):123-137, 2001.

[20] F.-X. Le Dimet. Une étude générale d'analyse objective variationnelle des champs météorologiques. Technical Report LAMP 28, Universite de Clermond II, BP 45, 63170, Aubiére, France, 1980.

[21] F.X. Le-Dimet and O. Talagrand. Variational algorithms for analysis and assimilation of meteorological observations. Tellus A, 38:97-110, 1986.

[22] F. X. LeDimet, I. M. Navon, and D.N. Daescu. Second order information in data assimilation. Monthly Weather Review, 130(3):629-648, 2002.

[23] Y. Li, I. M. Navon, P. Courtier, and P. Gauthier. Variational data assimilation with a semi-Lagrangian semiimplicit global shallow water equation model and its adjoint. Monthly Weather Review, 121(6):1759-1769, 1993.

[24] J. L. Lions. Optimal Control of Systems Governed by Partial Differential Equations. Springer Verlag, 1971.

[25] Lorenz. Predictabilty A problem partly solved. European Centre for Medium-Range Weather Forecasting, Shinfield Park, Reading UK.

[26] Hamill. T. M. and C. Snyder. A hybrid ensemble Kalman filter-3D variational analysis scheme. Monthly Weather Review, 128:2905-2919, 2000.

[27] P. Malanotte-Rizzoli. Modern Approaches to Data Assimilation in Ocean Modeling. Elsevier, Amsterdam, 1996.

[28] I. M. Navon, X. Zou, J. Derber, and J. Sela. Variational data assimilation with an adiabatic version of the NMC Spectral Model. Monthly Weather Review, 120(7):1433-1446, 1992.

[29] P P. Courtier, E. Anderson, W. Heckley, J. Pailleux, D. Vasiljevic, J. Hamrud, A. Hollingsworth, F.Rabier, and M.Fisher. The ECMWF implementation of three dimensional variational assimilation (3D-Var) I: Formulation. Quarterly Journal of Royal Meterology Society, 124:1783-1807, 1998.

[30] D. F. Parrish and J. C. Derber. The national meteological center's spectral statistical interpolation analysis system. Monthly Weather Review, 120:1747-1763, 1992.

[31] Arulampalam. M. S., Maskell. S., Gordon. N. J., and Clapp. T. A tutorial on particle filters for online nonlinear/non-Gaussian Bayesian tracking. IEEE transaction on signal processing, 150(2):174-188, 2002.

[32] Adrian Sandu and Lin Zhang. Discrete second order adjoints in atmospheric chemical transport modeling. Journal of Computational Physics, 227(12):5949-5983, 2008.

[33] Y.-K. Sasaki. An objective analysis based on the variational method. Journal of the Meteorological Society of Japan, II(36):77-88, 1958.

[34] S.E. Cohn S.E, D. M. Da Silva, J. Guo, M. Sienkiewiez, and D. Lamich. Assessing the effects of data selection with the DAO physical space statistical analysis system. Monthly Weather Review, 126:2913-2926, 1998.

[35] O. Talagrand and P. Courtier. Variational assimilation of meteorological observations with the adjoint equations Part I: Theory. Quarterly Journal of Royal Meterology Society, 113:1311-1328, 1987.

[36] J.-N. Thépaut and P. Courtier. Four-dimensional data assimilation using the adjoint of multi-level primitive equation model. Quarterly Journal of Royal Meterology Society, 117:1225-1254, 1991.

[37] Zhi Wang, I. M. Navon, F. X. LeDimet, and X. Zou. The second order adjoint analysis: Theory and applications. Meteorology and Atmospheric Physics, 50(1-3):3-20, 1992.

[38] M. Zhang, F. Zhang, and J.Hansen. Coupling ensemble Kalman filter with four-dimensional variational data assimilation. 22nd Conference on Weather Analysis and Forecasting/18th Conference on Numerical Weather Prediction, Park City, 2007.

[39] Ciyou Zhu, Richard H. Byrd, Peihuang Lu, and Jorge Nocedal. Algorithm 778: L-BFGS-B: Fortran subroutines for large-scale bound-constrained optimization. ACM Transactions on Mathematical Software, 23(4):550-560, 1997.

[40] X. Zou, I. M. Navon, and J. Sela. Variational data assimilation with moist threshold processes using the NMC spectral model. Tellus A, 45A:370-387, 1993. 


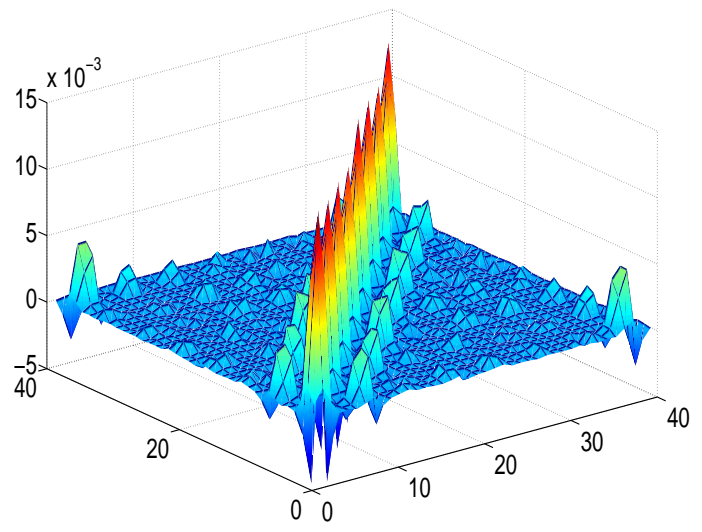

(a) Background covariance matrix at $t_{0}$

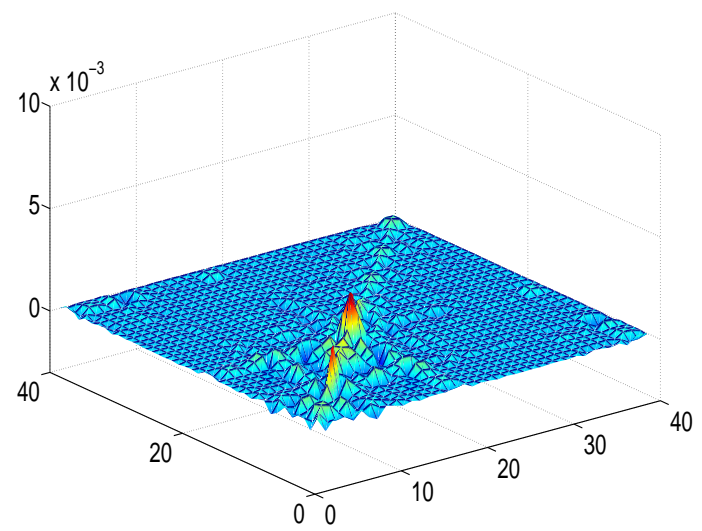

(c) Analysis covariance matrix at $t_{0}$

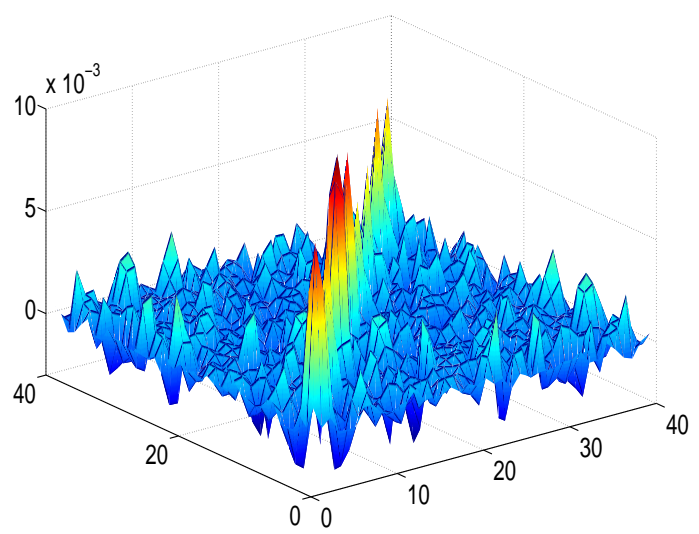

(e) Hybrid covariance matrix at $t_{0}$

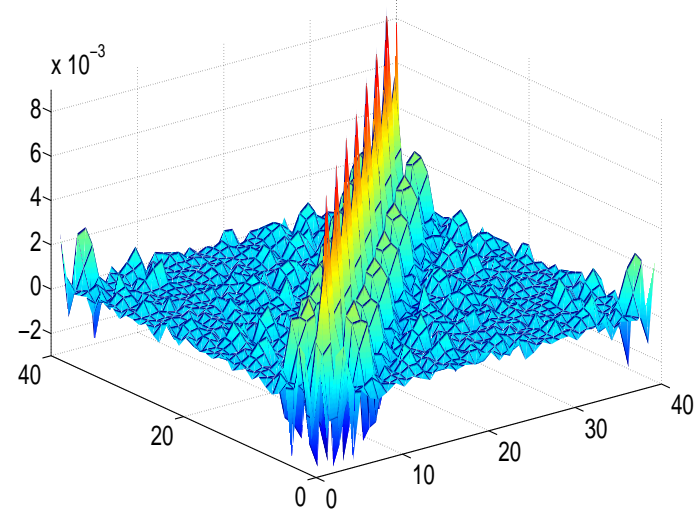

(b) Background covariance matrix at $t_{1}$

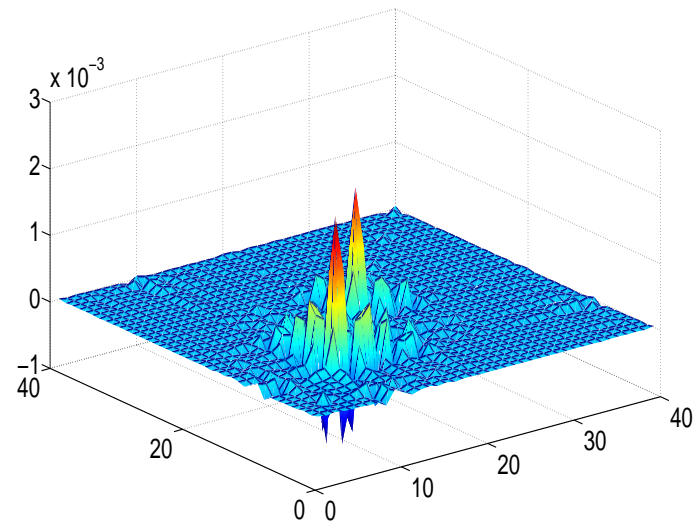

(d) Analysis covariance matrix at $t_{1}$

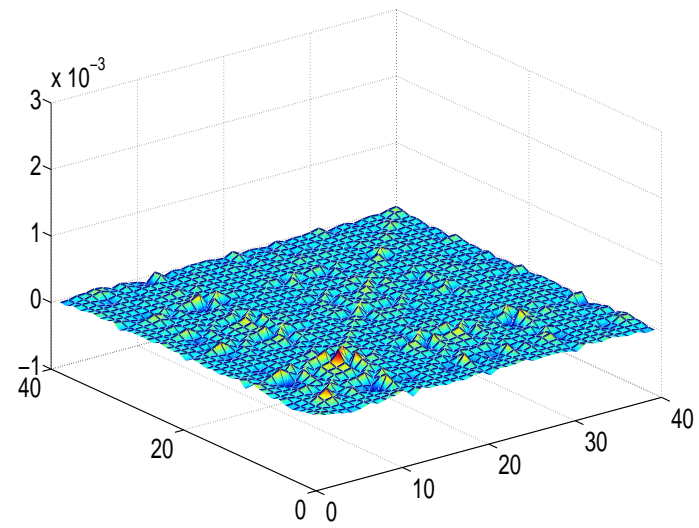

(f) Hybrid covariance matrix at $t_{1}$

Figure 1: Background (a-b), analysis reference $(\mathrm{c}-\mathrm{d})$, and hybrid $(\mathrm{e}-\mathrm{f})$ covariance matrix approximations at $t_{1}=0$ and $t_{2}=1$. 


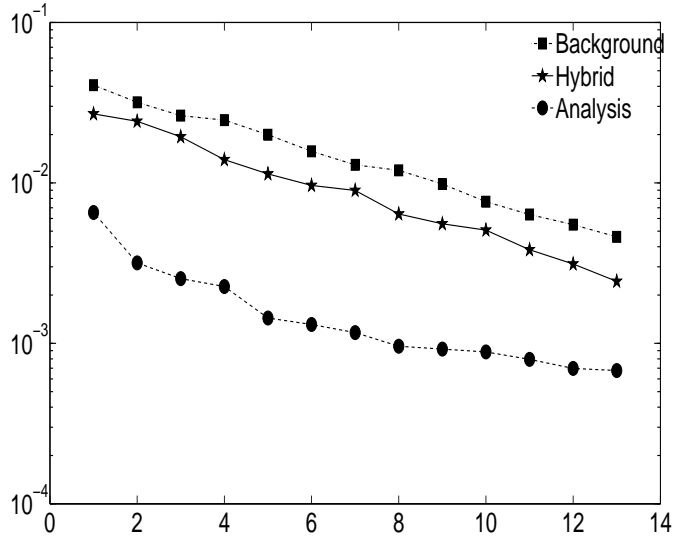

(a) Eigenvalues at time $t_{0}$.

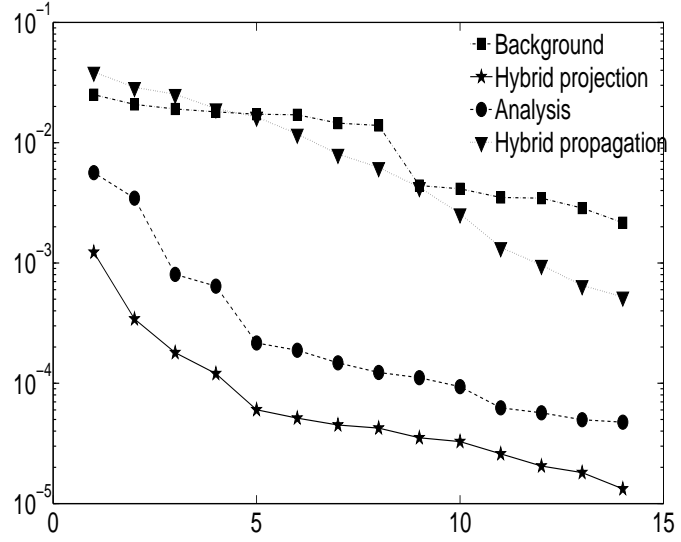

(b) Eigenvalues at time $t_{1}$.

Figure 2: Eigenvalue comparison of the background, analysis, and hybrid covariances at $t_{0}$ and $t_{1}$. The hybrid covariance at $t_{1}$ was computed through (i) projection out of the $4 \mathrm{D}$-Var increment subspace, and (ii) propagation of the hybrid ensemble members through the model from $t_{0}$ to $t_{1}$. 


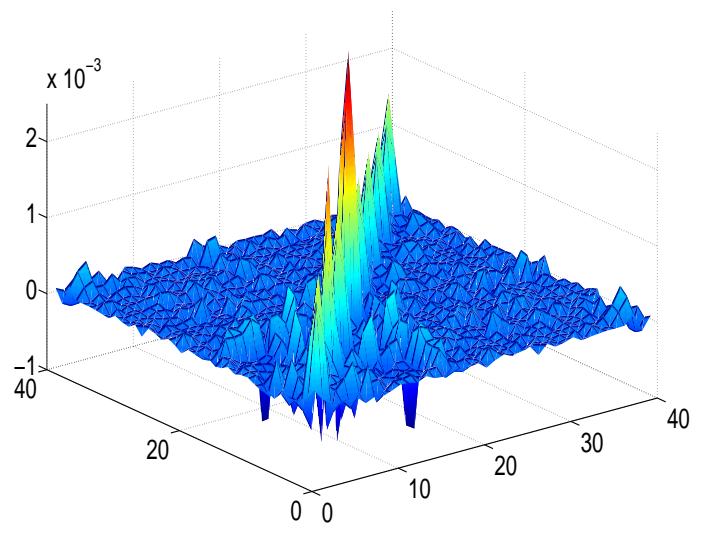

(a) Background covariance matrix at $t_{1}$

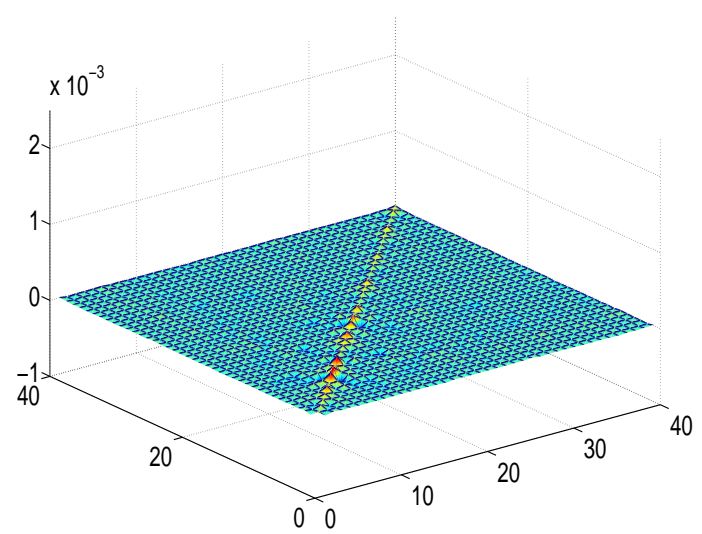

(c) Analysis covariance matrix at $t_{1}$

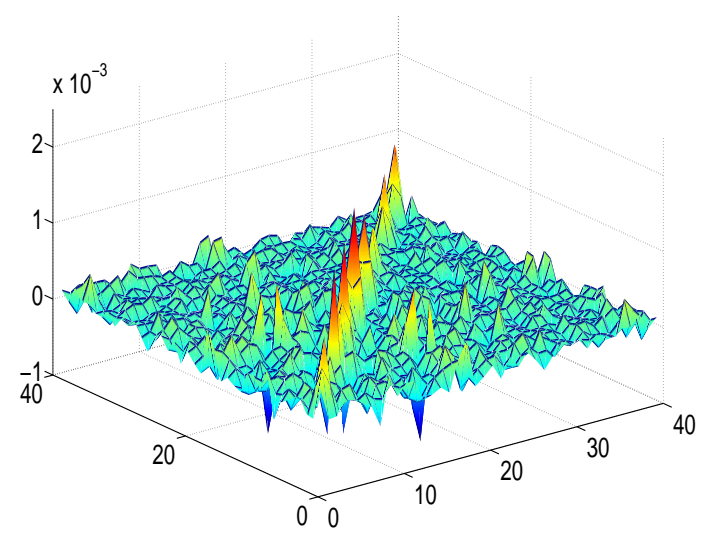

(e) Hybrid covariance matrix at $t_{1}$

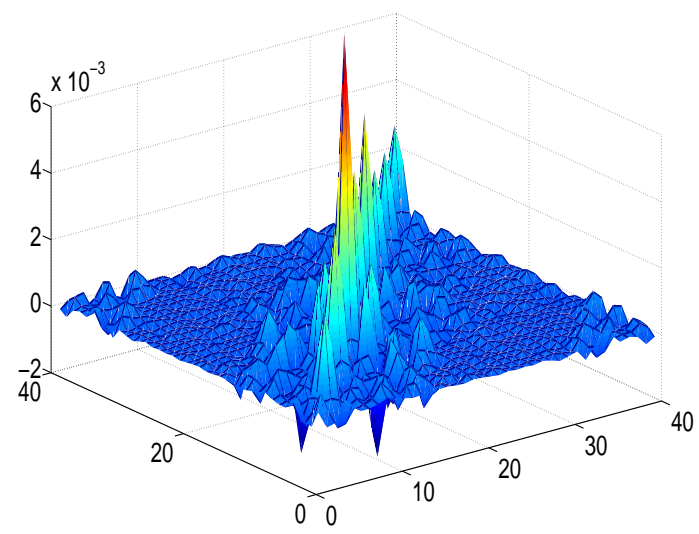

(b) Background covariance matrix at $t_{2}$

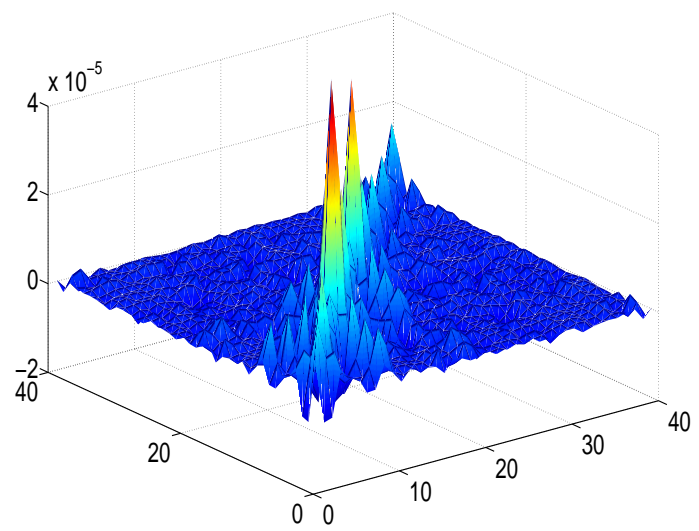

(d) Analysis covariance matrix at $t_{2}$

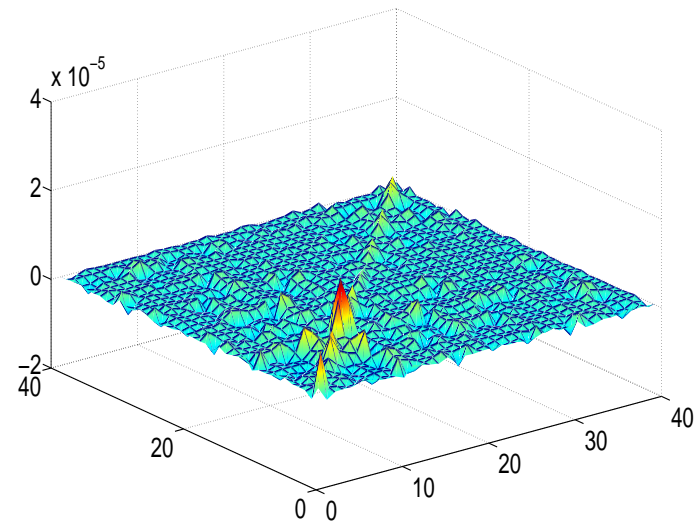

(f) Hybrid covariance matrix at $t_{2}$

Figure 3: Background (a-b), analysis reference $(\mathrm{c}-\mathrm{d})$, and hybrid $(\mathrm{e}-\mathrm{f})$ covariance matrix approximations at $t_{1}=1$ and $t_{2}=2$. 


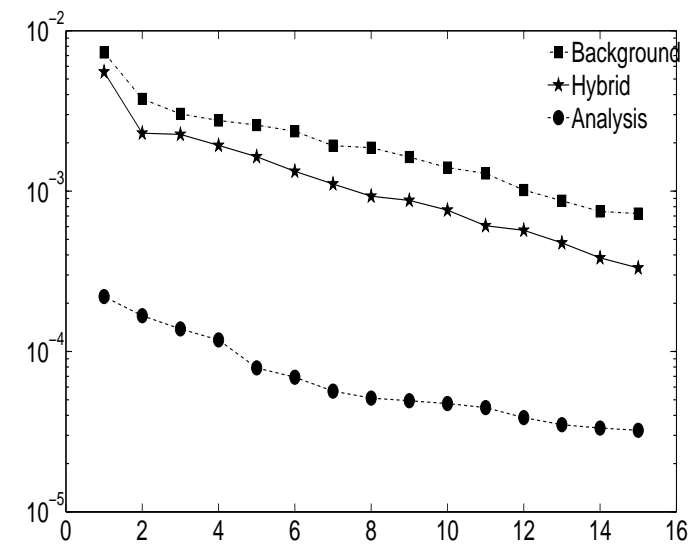

(a) Eigenvalues at time $t_{1}$

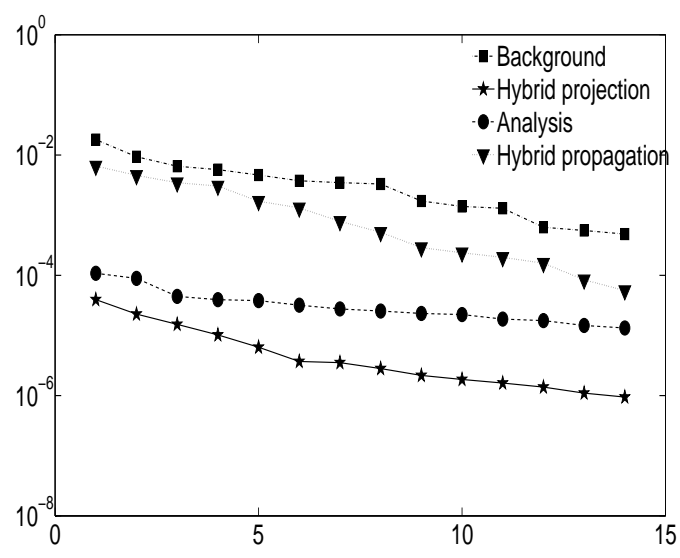

(b) Eigenvalues at time $t_{2}$

Figure 4: Eigenvalue comparison of the background, analysis, and hybrid covariances at time $t_{1}$ and $t_{2}$ (second assimilation window). 